\title{
Polypharmacy and high-dose antipsychotic regimes in the community ${ }^{\dagger}$
}

\author{
Tongeji E. Tungaraza, ${ }^{1}$ Seema Gupta, ${ }^{2}$ Jane Jones, ${ }^{2}$ Rob Poole, ${ }^{3}$ Gary Slegg ${ }^{4}$
}

The Psychiatrist (2010), 34, 44-46, doi: 10.1192/pb.bp.108.020776

${ }^{1}$ Wolverhampton PCT NHS Trust, Steps to Health, Wolverhampton; ${ }^{2}$ North East Wales NHS Trust,

Wrexham Maelor Hospital, Wrexham;

${ }^{3}$ Glyndŵr University, Wrexham;

${ }^{4}$ Section of Psychological Medicine,

Academic Unit, Wrexham

Correspondence to Tongeji $\mathrm{E}$.

Tungaraza (eliphaz@doctors.org.uk)

\begin{abstract}
Aims and method To determine the pattern of psychotropic prescribing in a group of people with psychosis who were living in the community under community mental health team (CMHT) care. Case-note entries over the previous 12 months were examined.
\end{abstract}

Results Only a third of individuals were on one psychotropic medication. Atypical antipsychotics were prescribed to $80.6 \%$. Polypharmacy was common. A third of people were taking three or more psychotropic drugs and $13.7 \%$ were on high-dose regimes, mostly involving two atypical antipsychotics.

Clinical implications The use of atypicals has not eliminated polypharmacy or highdose antipsychotic regimes. Clinicians need to be aware of this long-standing problem.

Declaration of interest T.E.T. accepted sponsorship to attend conferences from Janssen-Cilag, Eli Lilly, Bristol-Myers Squibb and Otsuka Pharmaceuticals. R.P. has accepted speakers' fees from Lundbeck, Eli Lilly and Pfizer, and accepted sponsorship to attend conferences from Wyeth, Astra Zeneca and Eli Lilly.
Prescribing more than one antipsychotic medication remains a common practice in psychiatry and has been labelled psychiatry's 'dirty little secret'. ${ }^{1}$ It is estimated that up to $40 \%$ of people with schizophrenia take two or more antipsychotics. ${ }^{2}$ A survey of in-patients in $49 \mathrm{UK}$ mental health services showed that $50 \%$ were receiving two antipsychotics. ${ }^{3}$ A prevalence of $69 \%$ has been observed in Japan. ${ }^{4}$ One of the undesirable consequences of the use of combinations of antipsychotic drugs is high-dosage regimes.

Guidelines acknowledge that high doses of antipsychotic medications may have a limited role in the treatment of schizophrenia. ${ }^{5}$ However, the practice is strongly discouraged. ${ }^{6}$ The extent of the use of high-dose antipsychotic regimes varies depending on the patient group surveyed. An in-patient survey of UK hospitals ${ }^{3}$ showed that $10.4 \%$ of individuals were prescribed doses above British National Formulary (BNF) recommendations (http://bnf.org/bnf/). A rate of around $28 \%$ was observed in a survey involving four European countries. ${ }^{7}$

\section{Method}

Most previous studies of antipsychotic polypharmacy and high-dosage regimes have been hospital based. Our study concerns individuals living in the community. The study was conducted within five community mental health teams

†See editorial, pp. 41-43, and review article, pp. 58-62, this issue.
(CMHTs) in urban and rural parts of North East Wales. On average each CMHT had 274 patients. Data were gathered retrospectively. Case-note entries for the previous 12 months (September 2006 to August 2007) were examined. All prescriptions and dosages of psychotropic medications were recorded.

The aim of the study was to observe the pattern of prescribing of psychotropic drugs and to determine the extent of high dose and polypharmacy regimes for individuals with schizophrenia and related disorders and bipolar disorders who are under the care of a CMHT. Although the use of some medications may have been transitional, we did not attempt to determine the rationale behind such regimes.

High-dose antipsychotic use was determined using chlorpromazine equivalents $(\mathrm{CPZeq})^{8,9}$ and aggregated percentages of $B N F$ maximum doses. Anyone receiving over $1000 \mathrm{mg}$ of CPZep or above $100 \%$ aggregated $B N F$ percentage was considered to be on a high dose. The percentage calculation alone was used for risperidone longacting injection and zotepine.

\section{Results}

The case notes of 211 individuals were examined: $59.7 \%$ male and $40.3 \%$ female. Mean age was 46.8 years (s.d. $=14.5$, range 20-80). Using broadly defined clinical diagnosis, 188 (89.1\%) had schizophrenia (including schizoaffective disorder) and 23 (10.9\%) had affective psychosis, predominantly bipolar 
affective disorder. Individuals had a mean of five consultations with a psychiatrist in a year (s.d. $=3.6$, range 1-27). Consultant psychiatrists were directly involved in $82 \%$ of all such reviews.

\section{Polypharmacy}

Only $32.7 \%$ of individuals were taking one antipsychotic medication alone. The remaining $67.3 \%$ were taking a combination of psychotropic drugs, i.e. an antipsychotic alongside an antidepressant, mood stabiliser, benzodiazepine, antimuscarinic or hypnotic. Overall, $37 \%$ were taking two psychotropics, $16.1 \%$ were on three, $10.4 \%$ on four, $3.3 \%$ were on five. One patient $(0.5 \%)$ was taking six psychotropic drugs (Fig. 1). Nearly a third of people were receiving three or more psychotropic medications.

A total of 174 individuals $(82.5 \%)$ were taking one antipsychotic, whereas 37 (17.5\%) were taking two antipsychotics (Fig. 1). No one was receiving more than two antipsychotics. In total, 170 people (80.6\%) were receiving 'atypical' antipsychotics, 38 (18\%) were on 'typical' antipsychotics and three (1.4\%) were taking a mixture of the two types.

\section{High-dose antipsychotic utilisation}

Twenty-nine (13.7\%) individuals were on a high-dose antipsychotic regime. Calculation of CPZep identified 13 people and 26 were identified by the aggregated percentage method.

As a group, all 29 individuals on high-dose antipsychotics were on atypicals, with the exception of one person who was taking a typical-atypical combination. Twenty individuals were taking a combination of two atypical antipsychotics, with only nine receiving one atypical medication. Seventeen of the high-dose regimes had been started within the past 12 months, and twelve of these regimes had been in place for over a year.

High-dose antipsychotic regimes were associated with taking two antipsychotics $(P<0.0001$, Fisher's exact test); receiving atypicals $(P<0.003$, Fisher's exact test); with being on antidepressants $(P<0.015$, Fisher's exact test); and with receiving three psychotropics or more $(P<0.0001$, Fisher's exact test) (Table 1). There was no association between high doses and gender $(P<0.067$, Fisher's exact test), although there was a trend towards being male. No association was observed between the use of mood stabilisers, benzodiazepines or antimuscarinics and highdose antipsychotic prescribing.

\section{Other psychotropics}

Antidepressants were being prescribed to $43.6 \%$ of the individuals, with $3.8 \%$ receiving two antidepressants. However, $54.5 \%$ of people had received antidepressants at some time in the previous 12 months. Mood stabilisers were prescribed to $14.7 \%$ of individuals. Lithium carbonate was prescribed to $5.7 \%$ of people and sodium valproate was prescribed to $6.1 \%$. Twenty-two (10.4\%) of individuals were receiving benzodiazepines, mostly commonly diazepam. Thirty-seven (17.5\%) were receiving antimuscarinic drugs

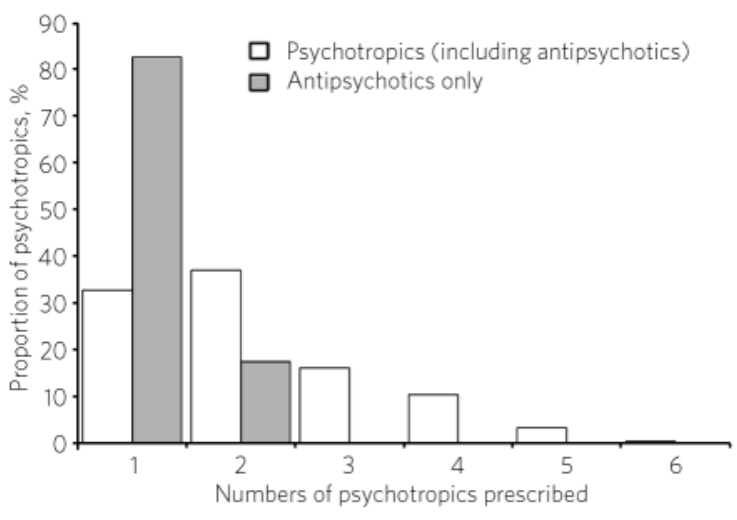

Fig 1 Polypharmacy prescribing pattern $(n=211)$.

and fifteen (7.1\%) were receiving hypnotics, mainly zopiclone.

\section{Discussion}

We observed a high rate of atypical antipsychotic use in CMHT patients. Our study shows that polypharmacy remains common, even among individuals who are settled and are resident in the community. Irrational regimes, such as atypical-typical antipsychotic combinations, were uncommon compared with previous in-patient studies.?

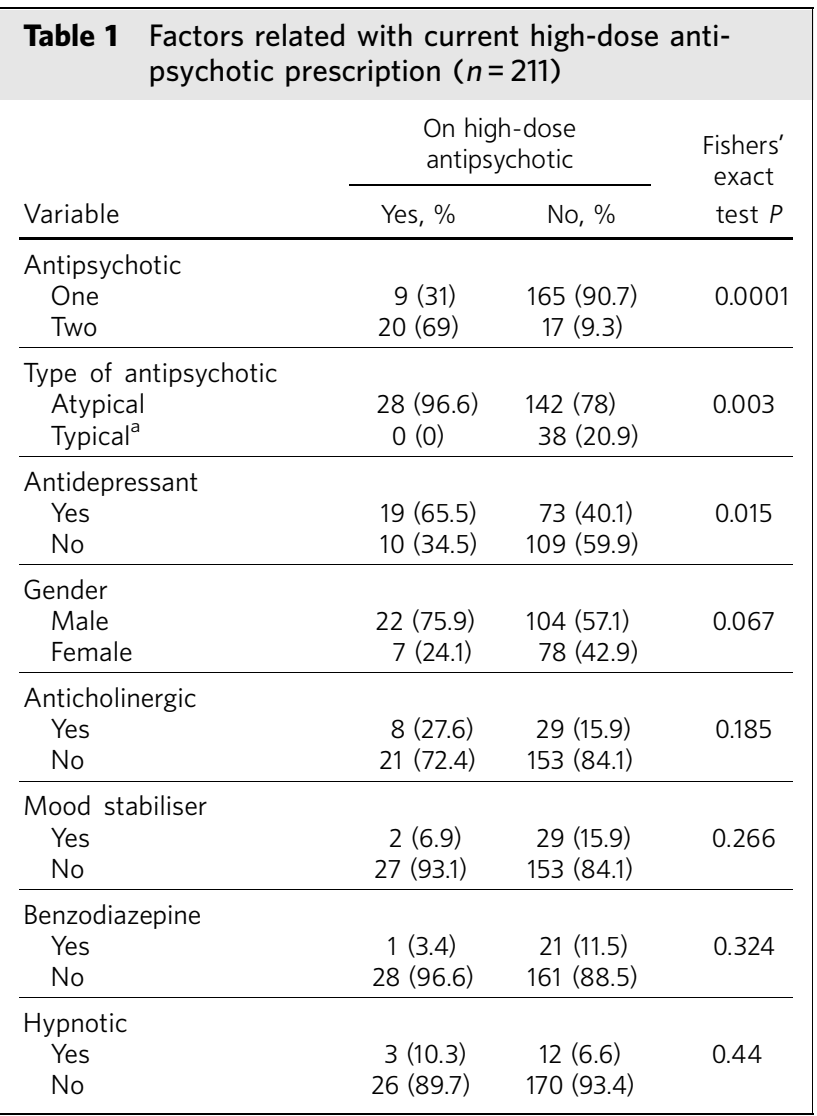

a. Three individuals were taking a mixture of typical-atypical antipsychotics and were not included. 
In accordance with National Institute for Health and Clinical Excellence (NICE) guidance, ${ }^{5}$ atypical anti-psychotics are recommended to be the first-line drug treatment for psychosis. Recent findings, ${ }^{10,11}$ although suggesting that they are no more effective and no better tolerated than typical antipsychotics, were probably too recent to have altered prescribing. The routine use of atypicals does not seem to have eliminated high-dose antipsychotic regimes, prescribed here for $13.7 \%$ of individuals. Our findings confirm the association between polypharmacy and highdose antipsychotic regimes. Two-thirds of high-dose regimes involved two atypicals in contrast to earlier findings where high-dose typical-atypical combinations were more common. $^{12}$

Almost a half of high-dose antipsychotic regimes had been followed for more than a year. Most of these individuals were on more than one antipsychotic and they were more likely to be receiving three or more psychotropics. It is possible that the prescribing clinicians were not aware that they were pursuing a high-dose regime. Persistent high-dose regimes carry risks for patients. ${ }^{13}$ Prescribers should be aware of the risk of high dosage when they prescribe drug combinations.

Our study has some limitations. It is based on retrospective examination of case notes. However, the quality of documentation was good. Letters to the general practitioner followed each consultation. Both the CPZep and the $B N F$ percentage methods of ascertaining high-dose regimes are imperfect, particularly as they aggregate doses of drugs with different mechanisms of action. However, they are well recognised and accepted methods that have been used elsewhere. ${ }^{6,12}$

Our study shows that people in the community are routinely exposed to polypharmacy and high-dose antipsychotic regimes. This is in contrast with guidelines and advice that have been available for many years suggesting that such regimes are unnecessary and potentially hazardous. Although attempts have been made to understand this phenomenon, ${ }^{4,14,15}$ little is understood about psychiatrists' prescribing behaviour and the reasons for it. In the light of the emergence of a new group of non-medical prescribers, there is an urgent need to understand how and why suboptimal prescribing occurs.

\section{About the authors}

Tongeji E. Tungaraza is Consultant Psychiatrist, Wolverhampton PCT NHS Trust, Steps to Health, Wolverhampton. Seema Gupta is Specialist Registrar in Psychiatry at Wrexham Maelor Hospital, Wrexham. Jane Jones works for
North East Wales NHS Trust at Wrexham Maelor Hospital, Wrexham. Rob Poole is Professor of Mental Health, Glyndŵr University, Wrexham. Gary Slegg is Research Fellow at the Section of Psychological Medicine, Academic Unit, Wrexham.

\section{References}

1 Stahl SM. Antipsychotic polypharmacy. Part 1: Therapeutic option or dirty little secret? J Clin Psychiatry 1999; 60: 425-6.

2 Canales PL, Olsen J, Miller AL, Crismon ML. The role of antipsychotic polypharmacotherapy in the treatment of schizophrenia. CNS Drugs 1999; 12: 179-88.

3 Lelliott P, Paton C, Harrington M, Konsolaki M, Sensky T, Okocha C. The influence of patient variables on polypharmacy and combined high dose of antipsychotic drugs prescribed for in-patients. Psychiatr Bull 2002; 26: $411-4$

4 Ito H, Koyama A, Higuchi T. Polypharmacy and excessive dosing: psychiatrists' perceptions of antipsychotic drug prescription. $\mathrm{Br} j$ Psychiatry 2005; 187: 243-7.

5 National Institute for Health and Clinical Excellence. Schizophrenia: Core Interventions in the Treatment and Management of Schizophrenia in Primary and Secondary Care. NICE, 2002.

6 Royal College of Psychiatrists. Consensus Statement on High Dose Antipsychotic Medication (Council Report CR138). Royal College of Psychiatrists, 2006.

7 Barbui C, Nosè M, Mazzi MA, Thornicroft G, Schene A, Becker T, et al. Persistence with polypharmacy and excessive dosing in patients with schizophrenia treated in four European countries. Int Clin Pharmacol 2006; 21: 355-62.

8 Jensen B. Antipsychotic comparison chart. RxFiles, 2003 (http://meds. queensu.ca/ clpsych/orientation/Antipschotics\%20Comparison\%20 Chart.pdf).

9 Taylor D, Paton C, Kerwin R. The Maudsley Prescribing Guidelines (8th edn). Taylor \& Francis, 2006

10 Lieberman JA, Stroup TS, McEvoy JP, Swartz MS, Rosenheck RA, Perkins $D O$, et al. Effectiveness of antipsychotic drugs in patients with chronic schizophrenia. N Engl J Med 2005; 353: 1209-23.

11 Jones PB, Barnes TRE, Davies L, Dunn G, Lloyd H, Hayhurst KP, et al. Randomized controlled trial of the effect on quality of life of second- vs first-generation antipsychotic drugs in schizophrenia: cost utility of the latest antipsychotic drugs in schizophrenia study (CUtLASS 1). Arch Gen Psychiatry 2006; 63: 1079-87.

12 Centorrino F, Eakin M, Bahk W, Kelleher JP, Goren J, Salvatore P, et al Inpatient antipsychotic drug use in 1998, 1993, and 1989. Am J Psychiatry 2002; 159: 1932-5.

13 Freudenreich O, Goff DC. Antipsychotic combination therapy in schizophrenia. A review of efficacy and risks of current combinations. Acta Psychiatr Scand 2002; 106: 323-30.

14 Tapp A, Wood AE, Secrest L, Erdmann J, Cubberley L, Kilzieh N. Combination antipsychotic therapy in clinical practice. Psychiatr Serv 2003; 54: 55-9.

15 Sernyak MJ, Rosenheck R. Clinicians' reasons for antipsychotic coprescribing. J Clin Psychiatry 2004; 65: 1597-1600. 